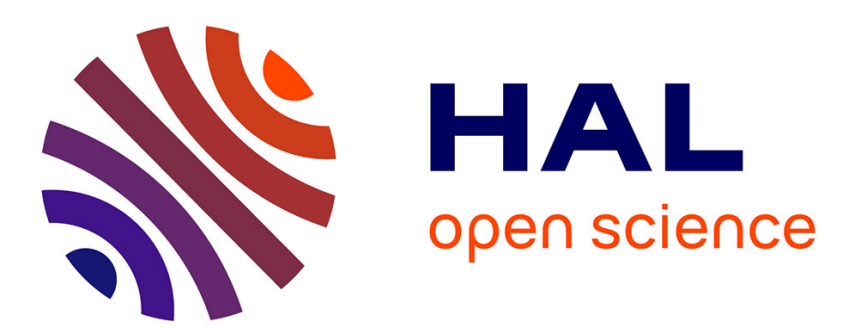

\title{
Temporal integration for amplitude modulation in childhood: Interaction between internal noise and memory
}

Laurianne Cabrera, Irene Lorenzini, Stuart Rosen, Léo Varnet, Christian Lorenzi

\section{To cite this version:}

Laurianne Cabrera, Irene Lorenzini, Stuart Rosen, Léo Varnet, Christian Lorenzi. Temporal integration for amplitude modulation in childhood: Interaction between internal noise and memory. Hearing Research, 2022, 415, pp.108403. 10.1016/j.heares.2021.108403 . hal-03469592

\section{HAL Id: hal-03469592 https://hal.science/hal-03469592}

Submitted on 7 Dec 2021

HAL is a multi-disciplinary open access archive for the deposit and dissemination of scientific research documents, whether they are published or not. The documents may come from teaching and research institutions in France or abroad, or from public or private research centers.
L'archive ouverte pluridisciplinaire HAL, est destinée au dépôt et à la diffusion de documents scientifiques de niveau recherche, publiés ou non, émanant des établissements d'enseignement et de recherche français ou étrangers, des laboratoires publics ou privés. 


\title{
Temporal integration for amplitude modulation in childhood: \\ Interaction between internal noise and memory
}

\author{
Laurianne Cabrera ${ }^{1,2}$, Irene Lorenzini ${ }^{1}$, Stuart Rosen ${ }^{2}$, \\ Léo Varnet ${ }^{3}$, Christian Lorenzi ${ }^{3}$
}

\footnotetext{
${ }^{1}$ Université de Paris, INCC UMR 8002, CNRS, F-75006 Paris, France

2 Speech, Hearing and Phonetic Sciences, UCL, UK

${ }^{3}$ Laboratoire des Systèmes Perceptifs (UMR 8248), CNRS, Ecole normale supérieure, Université Paris Sciences \& Lettres (PSL), Paris, France
}

Corresponding Author: Laurianne Cabrera, PhD

Integrative Neuroscience and Cognition Center (UMR 8002)

Université de Paris-CNRS

45 rue des saints pères

75006, Paris

laurianne.cabrera@parisdescartes.fr

Paper accepted for publication at Hearing Research in November 2021 DOI 


\section{Abstract}

It is still unclear whether the gradual improvement in amplitude-modulation (AM) sensitivity typically found in children up to 10 years of age reflects an improvement in "processing efficiency" (the central ability to use information extracted by sensory mechanisms). This hypothesis was tested by evaluating temporal integration for AM, a capacity relying on memory and decision factors. This was achieved by measuring the effect of increasing the number of AM cycles (2 vs 8 ) on AM-detection thresholds for three groups of children aged from 5 to 11 years and a group of young adults. AMdetection thresholds were measured using a forced-choice procedure and sinusoidal AM (4 or $32 \mathrm{~Hz}$ rate) applied to a $1024-\mathrm{Hz}$ pure-tone carrier. All age groups demonstrated temporal integration for $\mathrm{AM}$ at both rates; that is, significant improvements in AM sensitivity with a higher number of AM cycles. However, an effect of age is observed as both 5-6 year olds and adults exhibited more temporal integration compared to 7-8 and 10-11 year olds at both rates. This difference is due to: (i) the 5-6 year olds displaying the worst thresholds with 2 AM cycles, but similar thresholds with 8 cycles compared to the 7-8 and 10-11 year olds, and, (ii) adults showing the best thresholds with 8 AM cycles but similar thresholds with 2 cycles compared to the 7-8 and 10-11 year olds. Computational modelling indicated that higher levels of internal noise combined with poorer short-term memory capacities in children accounted for the developmental trends. Improvement in processing efficiency may therefore account for the development of AM detection in childhood.

Keywords Childhood, amplitude modulation, temporal integration, internal noise, short-term memory. 


\section{INTRODUCTION}

Amplitude-modulation (AM) information represents the slow temporal variations in amplitude over time of incoming sounds. AM is essential for speech comprehension as slow modulations $(<5-8 \mathrm{~Hz})$ convey important linguistic information (Drullman et al., 1994a, 1994b; Rosen, 1992; Varnet et al., 2017). To date, only a few studies have assessed in children the development of the ability to detect and discriminate slow AM fluctuations. Nevertheless, a typical development of slow AM perception is likely to be a pre-requisite of typical auditory and linguistic development (Goswami et al., 2002; Lorenzi et al., 2000; Witton et al., 2002).

Improvement in AM detection sensitivity during childhood may reflect the maturation of sensory-processing mechanisms leading to better temporal resolution with age, implying a better ability to follow fast changes in AM over time. Conversely, it is also possible that such improvement could reflect development in "processing efficiency". This would posit that the temporal resolution of the auditory system is adult-like but the ability to make optimal use of the extracted sensory information is not. This latter hypothesis was suggested in a pioneering study by Hall and Grose (1994). They showed that auditory sensitivity to sinusoidal AM, as measured by detection thresholds, improves with age between 5 and 10 years, but that AM sensitivity was similarly affected by AM rate (ranging from 5 to $200 \mathrm{~Hz}$ ) at all ages. This suggests that sensory factors constraining temporal resolution are mature early on, as also suggested by studies with young infants (Walker et al., 2019). However, in a recent similar study, Buss et al. (2019) found evidence that temporal resolution may be poorer in children than in adults for low AM rates. In this study, 5-to-11-year-old children and adults had to detect a target sinusoidal AM applied to a $4300-\mathrm{Hz}$ pure-tone carrier at three AM rates: 16, 64, and $256 \mathrm{~Hz}$. The results replicated improvement in AM detection thresholds with age at higher AM rates, 64 and $256 \mathrm{~Hz}$, but this was less clear for the slower rate of $16 \mathrm{~Hz}$. In another study, Peter et al. (2014) assessed AM detection thresholds in 8 to 11 year olds, 12- to 17 year olds and young adults using broadband noise carriers and AM rates ranging from 4 to $128 \mathrm{~Hz}$. Although, they did not observe a significant interaction between age and modulation frequency, they observed slightly worse thresholds in 8- to 11-year-old children compared to older children for a $4 \mathrm{~Hz}$ modulation rate, but not at higher AM rates. 
Note though that Hall and Grose (1994), Peter et al., (2014) and Walker et al. (2019) used a stochastic (broadband noise) carrier whereas Buss et al. (2019) used a deterministic one (a pure tone). It is thus possible that the external variability in acoustic stimuli may influence AM detection thresholds in childhood, and that temporal resolution even at low rates might improve between 5 and 11 years.

Another way to assess the development of sensory-processing mechanisms is to explore susceptibility to AM masking. It is now commonly assumed that AM perception reflects the operation of a bank of modulation filters selectively tuned for AM rate (Biberger and Ewert, 2016; Dau et al., 1997a) that are implemented centrally in the auditory system (Liégeois-Chauvel et al., 2004). "AM masking” effects are observed in psychophysical tasks using either two simultaneous sounds, a target and a masker, that fluctuate at a similar $\mathrm{AM}$ rate, or when the target and masking $\mathrm{AM}$ are simultaneously presented and applied to the same carrier. In such tasks, an elevation of detection thresholds for the target $A M$ is observed, reflecting the selectivity of modulation filters for AM rate (Bacon and Grantham, 1989; Houtgast, 1989).

In an additional experiment, Buss et al. (2019) measured AM masking in children and adults. The results showed comparable effects of masker AM rate and a lack of interaction with age suggesting that modulation selectivity is mature by 5 years of age. Nevertheless, the overall effect of age on detection thresholds in the masked conditions indicates reduced efficiency in AM processing in childhood. Cabrera et al. (2019) explored further the development of AM masking in childhood and the role of "processing efficiency" (in the case of AM perception, the ability to make optimal use of the available temporal-modulation information at the output of modulation filters). It was hypothesized that poor AM detection thresholds in childhood may relate to higher levels of internal noise (neural variability at each level of the auditory system) and/or the use of sub-optimal decision strategies. In this study, detection thresholds were measured for slow sinusoidal AM (with a rate of 4,8 , or $32 \mathrm{~Hz}$ ) applied to carriers whose inherent random modulations exerted different amounts of AM masking. More specifically three carriers were used: a pure tone, a narrowband noise with small inherent random AM fluctuations and a narrowband noise with higher inherent random 
AM fluctuations. Results showed that between 5 and 11 years, AM detection thresholds improved and surprisingly, that susceptibility to AM masking caused by the inherent random AM fluctuations of the narrowband noise carriers increased with age. Computer simulations of an auditory model based on the modulation-filterbank concept was used to simulate 1) poor sensory processing by changing the degree of selectivity of modulation filters; 2) poor processing efficiency by varying levels of internal noise at the output of the modulation filters; or 3) poor processing efficiency by simulating a suboptimal decision strategy. The model reducing internal noise levels with age by a factor 10 better accounted for the observed developmental trends between 5 and 11 years. Altogether, the findings on AM masking suggest that at least some aspects of AM processing mature during childhood.

To explore further the development of processing efficiency for AM in childhood, the current study focused on a phenomenon known as "temporal integration" for AM cues. Several studies in adults have shown that AM detection improves with an increasing number of AM cycles (Dau et al., 1997b; Sheft and Yost, 1990; Viemeister, 1979; Wallaert et al., 2016, 2017, 2018). This so-called "temporal integration" is currently assumed to reflect high-level, i.e., central, decision processes involving "multiple looks" (Viemeister and Wakefield, 1991) or a template-matching process (Dau et al., 1997b). Consistent with this idea, electrophysiological work conducted on gerbils showed that the parietal cortex is required to temporally integrate AM information, and thus, accumulate sensory evidence for discrimination decisions. Yao et al. (2020) measured gerbils' discrimination abilities between 4 and $10 \mathrm{~Hz}$ AM modulated noises using a behavioural task. The stimulus duration varied from 100 to $2000 \mathrm{~ms}$ to assess the shortest stimulus duration for which animals could discriminate $A M$, reflecting integration time. Pharmacological inactivation of the parietal cortex was shown to increase minimum integration times. More specifically, when the excitatory projections from auditory cortex to parietal cortex were chemogenetically inactivated, the behavioural integration time was significantly reduced, showing the role of parietal cortex in temporal integration of AM cues.

A recent investigation of temporal integration conducted with adult human listeners combining a psychophysical and modelling approach indicates that for low AM rates (below $5 \mathrm{~Hz}$ ), temporal integration is also constrained by the limited capacity of short- 
term auditory memory (Wallaert et al., 2017, 2018). In these studies, AM detection thresholds are measured in adult listeners as a function of the number of AM cycles available in the stimuli. A computational model was used to simulate the thresholds implementing different sources of internal noise supposed to limit the performance when processing AM. An additive noise was introduced to limit intensity discrimination and AM sensitivity. An additive time-varying memory noise was introduced to simulate imperfect retention of temporal-envelope information in each observation interval. All internal noises were modeled as Gaussian noises, and added independently to the output of the bandpass AM filter in the model.

In children, previous studies used only fixed stimulus durations to assess the effect of different AM rates on detection thresholds. Peter et al. (2014) suggested that the slightly worse thresholds observed at low AM rates may relate to the low number of cycles available in the target signal when the stimulus duration is kept constant at 500 ms. In their study, the 4-Hz rate involved the lowest number of AM cycles, 2 cycles, compared to the other AM rates tested. In contrast, Lorenzi et al. (2000) varied the target-stimulus duration from 500 to $1500 \mathrm{~ms}$ for a $4 \mathrm{~Hz}$ modulation rate and observed similar temporal integration between adults and six children between 8 and 15 years, suggesting no improvement between childhood and adulthood.

The present study sought to measure temporal integration at two different AM rates in a large cohort of children aged between 5 and 11 years, in order to probe the integrity and maturation of memory and decision processes in the AM domain. The main goal of the present study was to: 1) evaluate the effect of increasing the number of AM cycles ( 2 vs 8 cycles) on detection thresholds between 5 and 11 years for two slow AM rates (4 and $32 \mathrm{~Hz}$ ) and, 2) use computational modelling to test whether AMdetection data in children were better simulated by changes in the characteristics of AM filtering, internal noise and/or short-term memory capacities. It was assumed that temporal integration for AM detection should reflect the operation of the late, decision stage of AM processing (Dau et al., 1997b). It was also assumed that at the lower AM rate $(4 \mathrm{~Hz})$ where stimuli are longer in duration, temporal integration should reflect the additional constraints imposed by the limited echoic memory buffer involved in AM 
processing (King et al., 2019; Wallaert et al., 2017, 2018). If all age groups are similarly affected by the number of AM cycles or by AM rates, this would indicate that temporal integration is developed by 5 years and that central aspects of processing efficiency related to short-term memory and decision making for AM processing are well developed in childhood. However, if younger and older children are affected differently by the number of $A M$ cycles and by AM rates, this would suggest that memory and decision mechanisms are still evolving and thus, that processing efficiency for AM continues to develop into childhood.

\section{METHODS}

\subsection{Participants}

Seventy-two children and 30 adults were included in this experiment (see also Cabrera et al., 2019). All participants reported typical cognitive development. Consent was obtained from parents and adult participants as approved by the university ethics committee. Adult participants received a monetary compensation for their time and children collected stickers on a science certificate to keep them motivated.

For both children and adults, absolute thresholds were assessed for both ears at octave frequencies between 0.25 and $8 \mathrm{kHz}$ prior to testing. Two participants (one child and one adult) were excluded because their absolute thresholds for pure tones were above the normal range ( $>20 \mathrm{~dB} \mathrm{HL}$ ).

An additional 9 children were tested but not included in the final sample: three were at floor levels of performance for more than the half of the conditions (all aged 56 years), one withdrew from the study, four were not at school during testing, and one was excluded because of experimental error. Four adults were not included in the final sample because of missing data due to experimental errors. Furthermore, an outlier labelling rule (Hoaglin and Iglewicz, 1987) was applied to the data set. A total of 10 outliers were flagged and their data were not included in the analyses (see Cabrera et al., 2019). Four outliers were flagged in the 5-6 year-old group, two in the 7-8 year olds, four in the $10-11$ year olds, and two in the adult group (11\% of the data). When those outliers were removed, the data did not differ from normality (KolmogorovSmirnov test, all ps > 0.05). 
The final sample included: 215 -6-year-olds ( 10 females; mean age $=5.7$ years, $\mathrm{SD}=0.4$ ), 27 7-8-year-olds ( 13 females; mean age $=7.8$ years, $S D=0.5$ ), 24 10-11year-olds ( 12 females; mean age $=10.7, \mathrm{SD}=0.4$ ) and 30 adults $(22$ females, mean age $=22.5$ years, $S D=2.5$ ).

\subsection{Stimuli}

A full factorial design led to four experimental conditions: two target AM rates $\left(f_{m}\right.$ : 4 and $32 \mathrm{~Hz}$ ) presented for two numbers of modulation cycles (2 and 8). As previous studies showed that optimal AM detection is observed with 4-5 cycles, we selected 8 cycles to ensure that listeners are presented with a sufficient number of cycles (Edwards et al., 2008; Lee and Bacon, 1997). In all conditions, the stimuli included 50$\mathrm{ms}$ raised-cosine onset/offset ramps, and the inter-stimulus interval was $500 \mathrm{~ms}$. Standard sounds were not modulated in amplitude, and target sounds were modulated at depths ranging from $m=100 \%$ to $m=1 \%$, in 20 steps of $2 \mathrm{~dB}$. The starting phase of the modulation was randomized on each trial. The carriers were sine tones centered at $1027 \mathrm{~Hz}$, generated with a random starting phase at a sampling frequency of 44.1 $\mathrm{kHz}$. Figure 1 shows the waveforms played in the 4 conditions. 


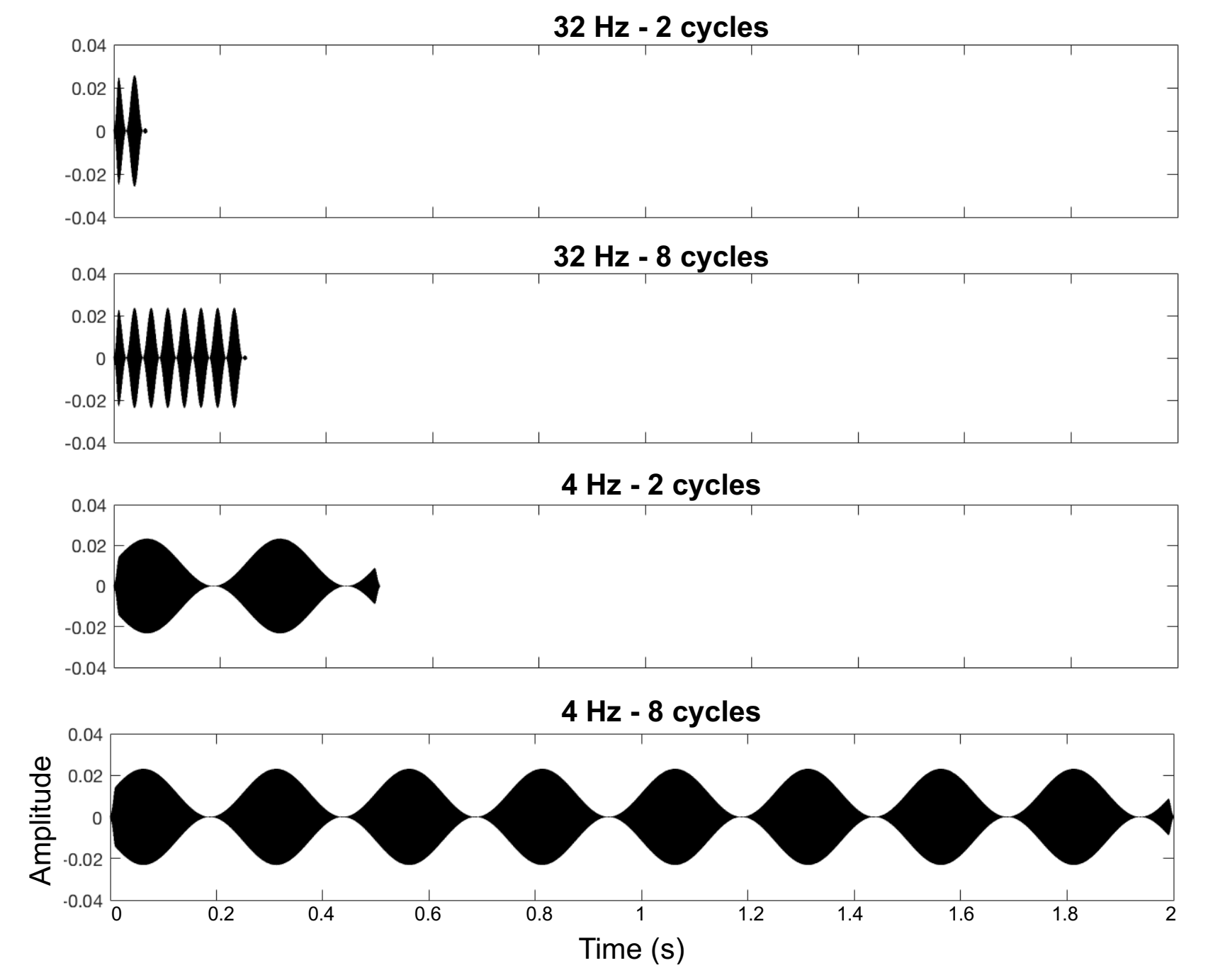

Figure 1. Waveforms of one stimulus exemplar in each condition. The target sounds modulated at $32 \mathrm{~Hz}$ were 62.5- and 250-ms long in the 2 and 8cycle conditions, respectively (first two upper rows). The target sounds modulated at $4 \mathrm{~Hz}$ were 500 - and 2000-ms long in the 2 and 8-cycle conditions, respectively (first two lower rows). 


\subsection{Procedure}

The present experiment was part of a larger project on temporal processing capacity in childhood assessing modulation masking as well (see Cabrera et al., 2019). Children completed the whole experiment within 4 sessions of 20 min over a 2week period and adults in one session of $1.5 \mathrm{hr}$. The 4 experimental conditions considered here occurred randomly during the whole experiment. AM detection thresholds were measured using a three-interval, three-alternative forced-choice (3l3AFC) adaptive procedure, implemented on a touch-screen tablet. The sounds were played diotically through headphones (Sennheiser HD 25-SP II) at a level of $65 \mathrm{~dB}$ SPL.

In this task, participants were asked to listen to three animal characters who would appear on the screen and produce a sound one after the other and to find the odd-one out, i.e., the modulated target. On each trial, participants had unlimited time to respond and received visual feedback as to the correctness of their response. The next trial started automatically $600 \mathrm{~ms}$ after the participant's response.

The response on the first trial was always ignored and a one-down, one-up rule was used until the first reversal (Baker and Rosen, 2001). Then, a 2-down 1-up adaptive procedure was used to track the $71 \%$ correct-point (Levitt, 1971). The first trial started at $m=100 \%$ and the initial step-size (the change in modulation depth) was $6 \mathrm{~dB}$. This step size was reduced to $4 \mathrm{~dB}$ after the first reversal, and to $2 \mathrm{~dB}$ after the second reversal, the minimum $m$ was $1 \%$. The run stopped after the 8 th reversal or after 32 trials. One estimate was collected for each participant in each condition and a second estimate was collected if fewer than 5 reversals were obtained, or if the track did not converge. The threshold in $\mathrm{dB}$ was the geometric mean of the last four reversals.

\section{RESULTS}

\subsection{AM detection thresholds (AMDTs)}

Figure 2 represents the AM-detection thresholds (AMDTs) according to the number of AM cycles and modulation rate. All age groups show lower (better) AM detection thresholds in the 8-cycle conditions compared to 2-cycle conditions. 
A mixed ANOVA assessed the effect of the within-subject factors of Cycle (2 levels: 2 and 8 cycles) and Rate (2 levels: 4 and $32 \mathrm{~Hz}$ ) and the between-subjects factor of Age (4 levels: 5-6 years, 7-8 years, 10-11 years and adults) on the AMDTs. The analysis revealed a significant main effect of Rate $\left[F(1,98)=13.34, p<.001, \eta^{2}=.12\right]$, Cycle $\left[F(1,98)=518.16, p<.001, \eta^{2}=.84\right]$, Age $\left[F(3,98)=13.13, p<.001, \eta^{2}=.29\right]$ and $a$ significant interaction between Cycle and $\operatorname{Age}\left[F(2,98)=5.60, p=.001, \eta^{2}=.15\right]$. Other comparisons were not significant [Rate $\times$ Age $F(3,98)=.34, p=.798$; Rate $\times$ Cycle $F(1,98)=1.03, p=.312 ;$ Rate $\times$ Cycle $\times$ Age $F(3,98)=.43, p=.735]$.

For the main Age effect, pairwise comparisons with Bonferroni corrections showed that, overall, 5-6 years have higher (worse) AMDTs than 7-8,10-11 years and adults ( $p s<.01)$. The groups of 7-8 years showed similar AMDTs than $10-11$ years $(p>.05)$ but worse AMDTs than adults $(p=.016)$. The group of $10-11$ years showed overall similar AMDT than the group of adults $(p=.11)$. Moreover, AMDTs were overall lower (better) at a high rate than at a low rate.

The analyses of the Cycle and Age interaction indicated that all age groups show temporal integration, that is, lower (better) AMDTs with increasing number of AM cycles (ps < .001). It is important to note that 5-6-year-olds showed worse AMDTs in the 2-cycle condition compared to the three other age groups $(p<.001)$, and that adults showed significant better AMDTs in the 8-cycle condition compared to all the other age groups $(p<.001)$. 


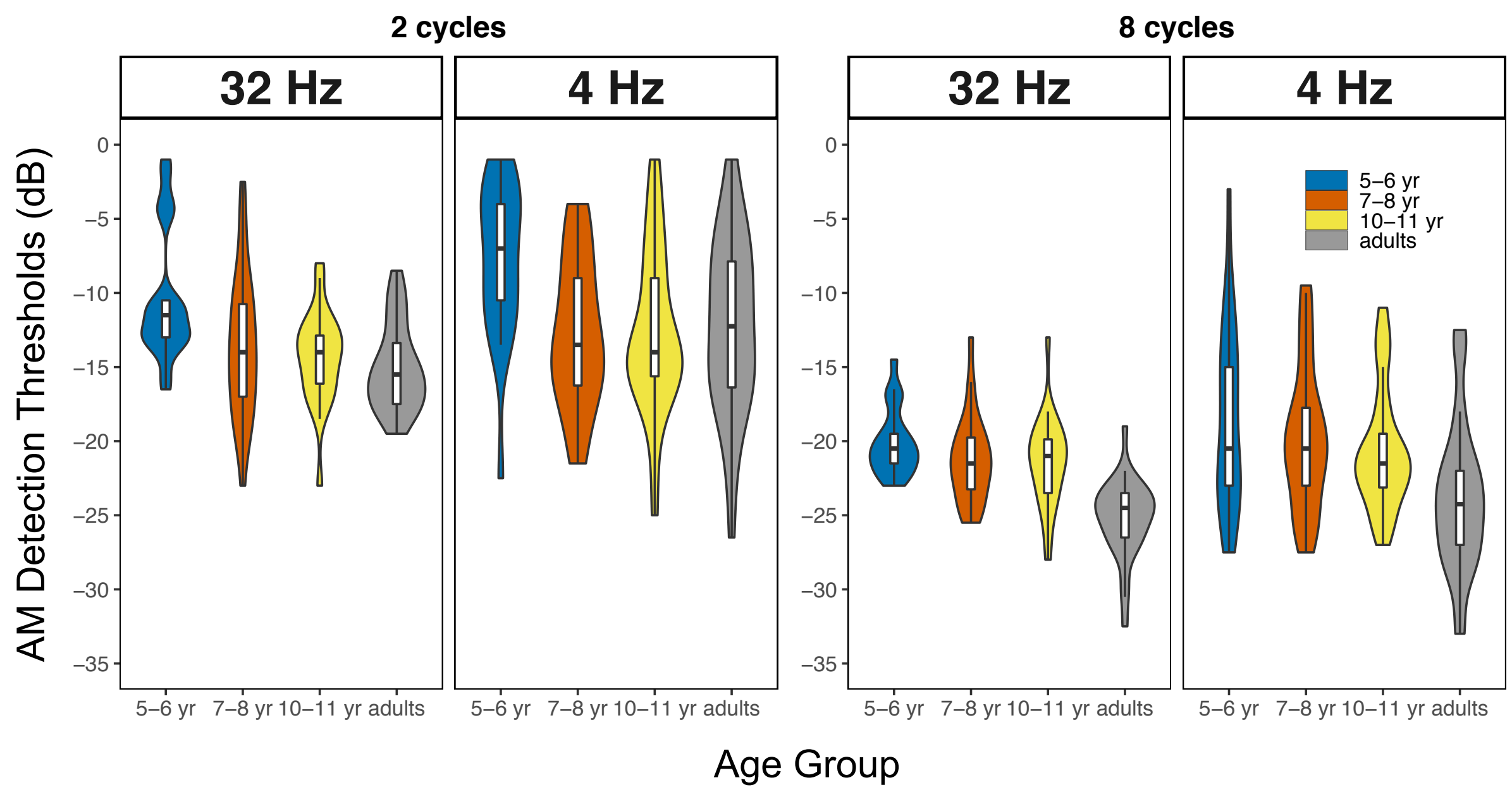

Figure 2. AMDTs (in $\mathrm{dB}$ ) for each age group. The left and right panels represent thresholds in the 2-cycle and 8-cycle conditions, respectively, at each modulation rate (4 and $32 \mathrm{~Hz}$ ). The boxes in the boxplots represent the 25th, 50th and 75th percentile for each group/condition, with whiskers extending to the full range of the data. The violin plots illustrate the kernel probability density (i.e. the width of the violin area represents the proportion of the data located there). 


\subsection{Temporal-integration effect}

A second ANOVA was run on the temporal integration scores shown in Figure 3, that are estimated as the difference in AMDTs between 2 and 8 cycle conditions, with Rate (2 levels) as a within-subject factor and Age (4 levels) a between-subject factor. This score represents the improvement in AMDTs when increasing the number of $A M$ cycles from 2 to 8 . The analysis revealed no significant effect of Rate $[F(1,98)$ $=1.03, p=.312]$, nor significant interaction between Rate and $\operatorname{Age}[F(2,98)=0.74, p$ $=.735]$, but a significant effect of Age $\left[F(3,98)=5.60, p=.001, \eta^{2}=.15\right]$. Further analyses of the Age effect showed that 5-6 years showed slightly more temporal integration than the 7-8 years and 10-11 years ( $p s=.085$ and .16 with Bonferroni corrections, respectively), but showed a similar level of integration to adults $(p=1)$. Interestingly, adults showed higher integration than the 7-8 years and 10-11 years ( $p$ $=.007$ and .015 , respectively). This is related to the worse AMDTs shown by the youngest group in the 2-cycle condition and to the best AMDTs observed in the 8cycle condition in adults, making these two groups exhibiting a larger difference between the 2 and 8 cycle conditions.

To sum up, all child groups showed significant effects of AM rate and AM cycle, but 5-6 years and adults exhibited similar levels of temporal integration for AM detection. Indeed, at both rates, the youngest age group showed significantly higher (worse) AMDTs in the 2-cycle condition and reached similar thresholds in the 8-cycle condition compared to the groups of 7-8 years and 10-11 years. Furthermore, adults outperformed all child groups in the 8-cycle conditions indicating that temporal integration continues to develop after 11 years of age. 


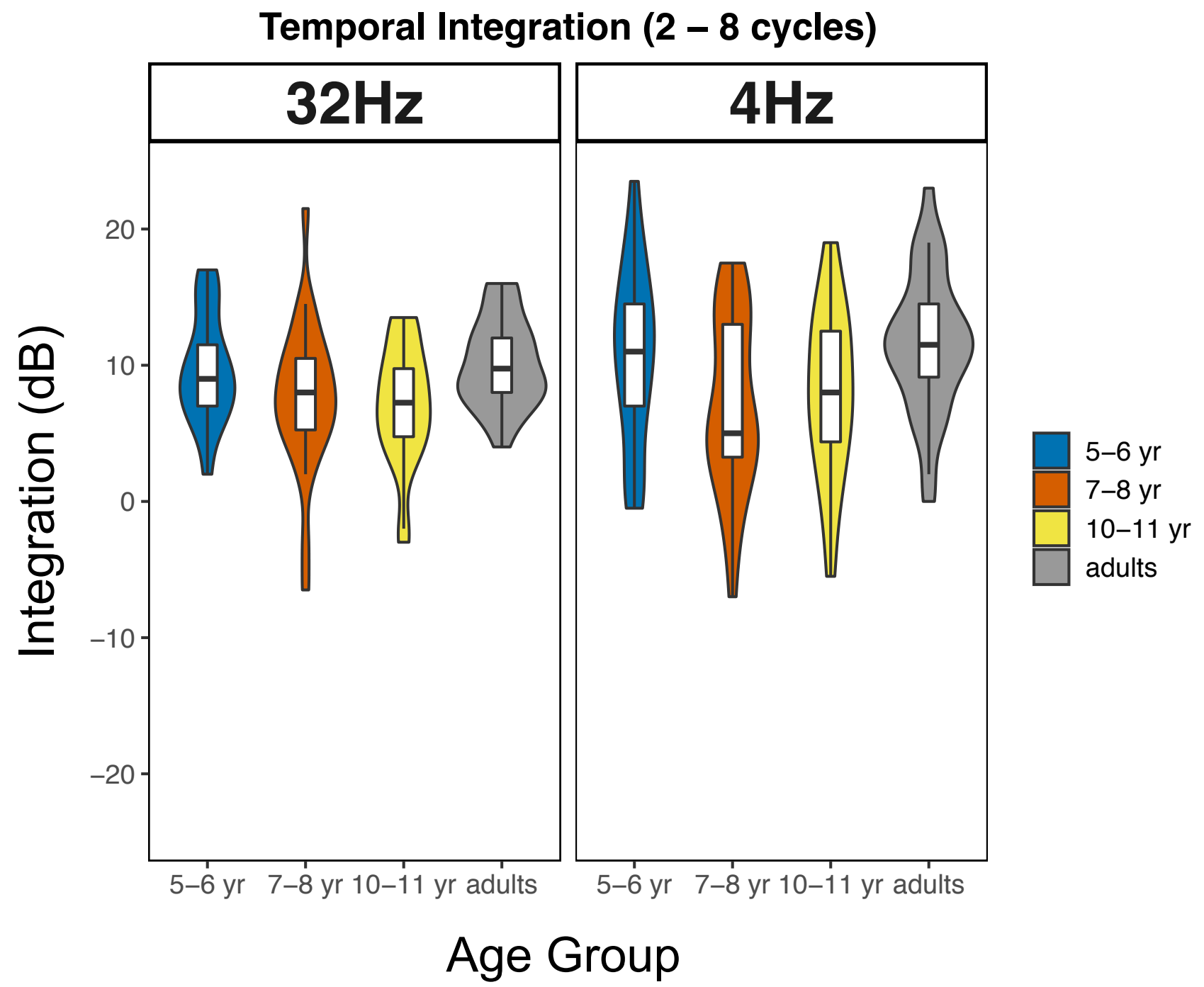

Figure 3. Integration scores (in $\mathrm{dB}$ ) for each age group. Box- and violin plots are as described for Figure 2. 


\subsection{Modelling study}

A computational model of AM processing was used to assess whether the differences between age groups could relate to changes in (low-level) sensory processing or in (high-level) processing efficiency. The same model as in Cabrera et al. (2019) was used to simulate AMDTs while manipulating parameters at different stages of AM processing. Three main processing stages were represented in this model: Early (i.e., peripheral) processing (bandpass cochlear filtering, instantaneous amplitude compression, half-wave rectification, short-term adaptation), mid-level processing (envelope extraction via a bank of selectively-tuned AM filters with a constant $Q$ value of 1 ), and late processing (additive internal noise; exponential memory noise with a 1.4-sec time constant, template-matching decision device). The model structure is detailed in the Appendix. The "late processing" stage implements central factors constraining processing efficiency, that is, the ability to make optimal use of the available AM information extracted by sensory mechanisms at early and mid-level stages. This model previously suggested that AM-masking data in childhood were better predicted by a drastic change in the additive internal noise between 5 and 11 years (by a factor 10 ).

\subsubsection{Effects of varying the magnitude of the additive internal noise}

In the current study, the first model, named Model $A$, uses the standard deviation (SD, in model units, m.u.) of the additive internal noise that best fitted the AMDT data of Cabrera et al. (2019). More precisely, Model A uses the SD of the internal noise that maximized the model's "goodness-of-fit" at the $8 \mathrm{~Hz}$ AM rate. This was achieved by minimizing the model's mean absolute error, evaluated as the average absolute value of the difference between simulated AMDTs and "real" AMDTs averaged across participants for each age group in Cabrera et al. (2019). The SD of the internal noise is set to $2 \times 10^{-4}$ for adults, $5 \times 10^{-4}$ for 10-11-year-olds, $1 \times 10^{-3}$, for 7-8-year-olds, and $2 \times 10^{-3}$ for 5-6- year-olds. So, the SD of internal noise varies by a factor 10 between 5-6-year-olds and adults. Real averaged data and simulated data are shown in Figure 4. As expected, Model A yielded better AMDTs with decreasing level of internal noise. At both AM rates, the improvement in AM sensitivity is larger for $8 \mathrm{AM}$ cycles $(\sim 5 \mathrm{~dB})$ than for $2 \mathrm{AM}$ cycles $(\sim 2 \mathrm{~dB})$, predicting increased temporal 
1 integration over age. However, Model A does not fit the real data well, specially for the

2 2-cycle and $32-\mathrm{Hz}$ condition, with the model prediction error averaged across age 3 groups $=4.7 \mathrm{~dB}$.

4 Model A reproduces well the integration data for the group of 5-6 years at 32

$5 \mathrm{~Hz}$ and $4 \mathrm{~Hz}$ but not for the other groups of children nor for the adult group specially 6 at $32 \mathrm{~Hz}$. According to this model, a decrease in the level of the additive internal noise 7 by a factor of 10 is associated with an increase in temporal integration by about $4 \mathrm{~dB}$ 8 at $4 \mathrm{~Hz}$ and $7 \mathrm{~dB}$ at $32 \mathrm{~Hz}$. In contrast with the simulated data, temporal integration 9 decreases in real listeners for intermediate age groups (7-6 years and 10-11 years). 10 The failure of Model $\mathrm{A}$ at $32 \mathrm{~Hz}$ relates partially to the short duration of the stimuli 11 when using 2 AM cycles $(62.5 \mathrm{~ms})$. In this case, the response of the simulated 12 modulation filter tuned to $32 \mathrm{~Hz}$ (target rate) is dominated by the response of the filter 13 to the stimulus onset (see Edwards et al., 2008; Sheft and Yost, 1990). The latter is 14 superimposed on the target and masks its temporal structure, resulting in poor detection of the AM fluctuation. Thus, the following paragraphs explore the role of two specific parameters related to the model's goodness of fit only for the 4-Hz conditions. 

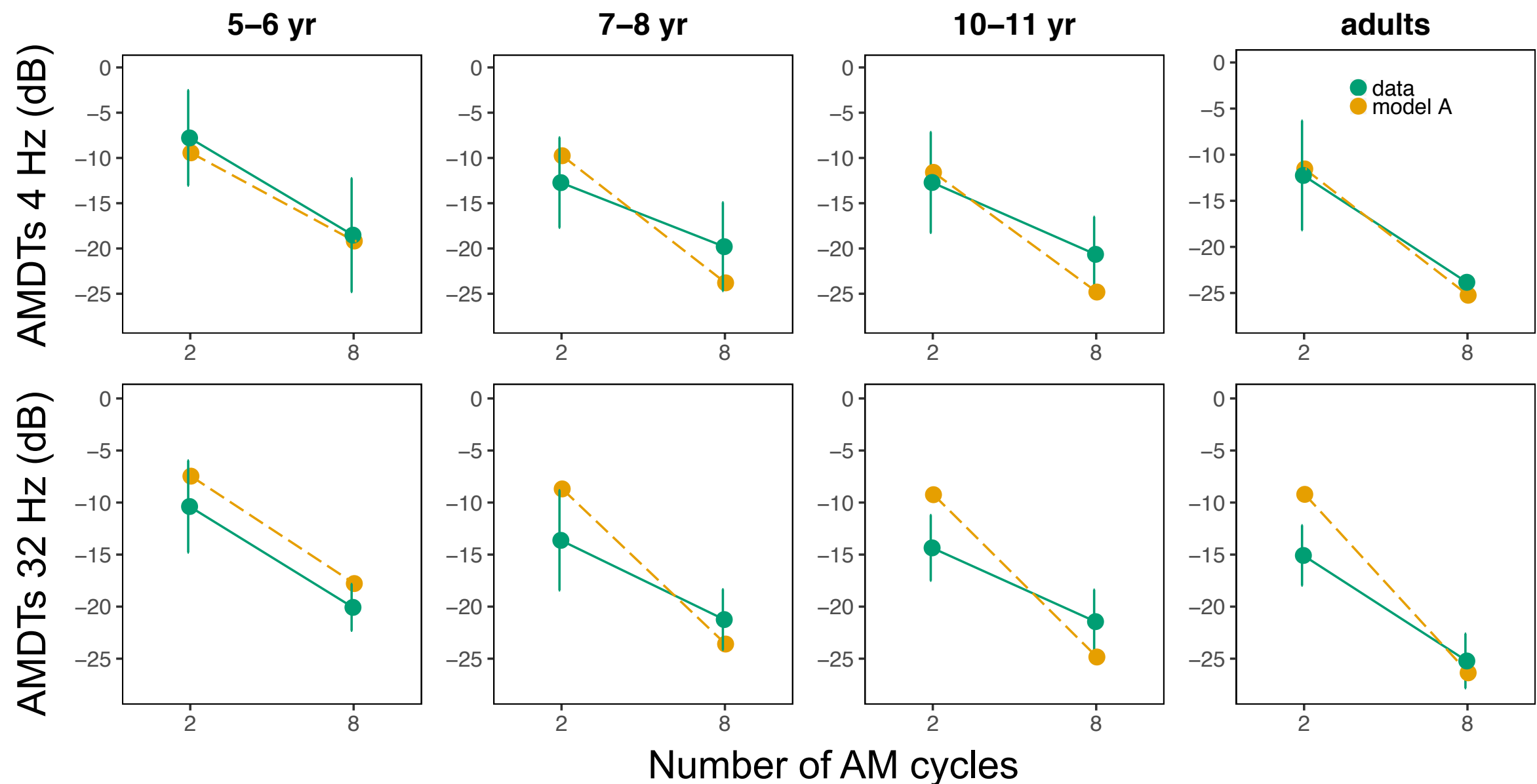

\section{Number of AM cycles}

1 Figure 4. Temporal integration effects: AMDTs (in dB) for each age group (from left to right column: 5-6 years, 7-8 years, 10-11 years 2 and adults) as a function of number of AM cycles (2 vs 8 cycles) at each modulation rate (4 Hz: upper row; $32 \mathrm{~Hz}$ : lower row). The 3 real data are represented with the green circles and solid lines. The simulated data with Model A are represented with the orange 4 circles and dashed lines. 


\subsubsection{Effects of varying the strength of short-term adaptation}

At the early and mid-level processing stages, two crucial aspects of temporal processing can be modified in the model: the highpass filtering of the compressed output of cochlear filters which simulates short-term adaptation effects in the temporalenvelope domain and AM selectivity as reflected in the bandwidths of the modulation filters. The effect of changing the latter parameter was tested in Cabrera et al. (2019). A progressive increase in AM selectivity (in other words, a reduction in the bandwidth of modulation filters) as a function of age did not account for real AM detection data. The effect of changing the short-term adaptation parameter is tested here.

In Model A, the cutoff frequency of the highpass filter (rolling off at $20 \mathrm{~dB}$ per decade) applied to the output of all peripheral (cochlear) channels is initially set to 2.5 $\mathrm{Hz}$ as in Wallaert et al. (2018; see also Tchorz and Kollmeier, 1999) to simulate shortterm adaptation effects observed in adult listeners. The cutoff frequency of the highpass filter is modified in Model B. Increasing the cutoff frequency of the highpass filter has the effect of enhancing the transient response of the model at the stimulus onset. Here, it is hypothesized that the youngest children may be more distracted by this transient signal than older children. For each AM rate, this "bottom-up" attentional effect would result in a greater detrimental effect on AM detection at the shortest stimulus duration (i.e., 2 cycles), increasing consequently temporal integration for AM detection. Although the current model was not designed to account for sustained attentional effects in AM perception, its decision stage based on a template-matching strategy (see Appendix section) could nevertheless be influenced by the presence of strong onset cues at the output of the simulated cochlear filters. The cutoff frequency of the highpass filter was increased from $2.5 \mathrm{~Hz}$ (Model A) to $3.5 \mathrm{~Hz}$ (Model B1) or decreased to $1.5 \mathrm{~Hz}$ (Model B2). As observed in Figure 5.a and 5.b, increasing the cutoff frequency of the highpass filter to $3.5 \mathrm{~Hz}$ (Model B1) has the effect of elevating AMDTs for the $4 \mathrm{~Hz}-2$ cycle condition for the groups 7-8 years and 10-11 years, by 1 and $2 \mathrm{~dB}$, respectively, making model predictions more consistent with the data (model fitting error $<1 \mathrm{~dB}$, Figure 5.b). However, for the $4 \mathrm{~Hz}-8$ cycle condition, changing the cutoff frequency of the highpass filter does not improve the fit for any group. Figure 5.c shows the effect of changing the adaptation parameter at the output of the adaptation filter of the model that influences slightly the transient response over the 
1 first $0.2 \mathrm{~s}$ of the stimulation. In this case, the model's fitting errors for the integration 2 effect are still greater than $4 \mathrm{~dB}$ for those two groups. Manipulating the characteristics 3 of the adaptation stage (data not shown) has little effect on the predicted AMDTs for 4 the $32-\mathrm{Hz}$ AM rate. These simulations suggest that children do not place more weight 5 on the onset of the signal or that this effect varies over age. Overall, the model using 6 a cutoff frequency of $2.5 \mathrm{~Hz}$ (Model A) gives a better account of real data for all groups, 7 suggesting that the effects of short-term adaptation (Models B1 and B2) do not differ 8 markedly over age.

9 

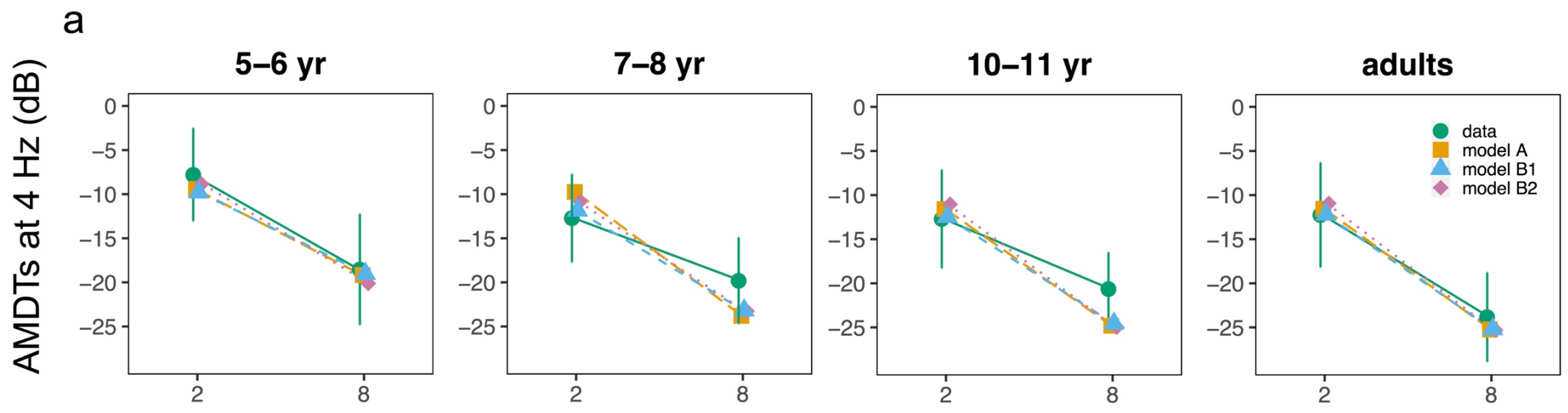

Number of AM cycles
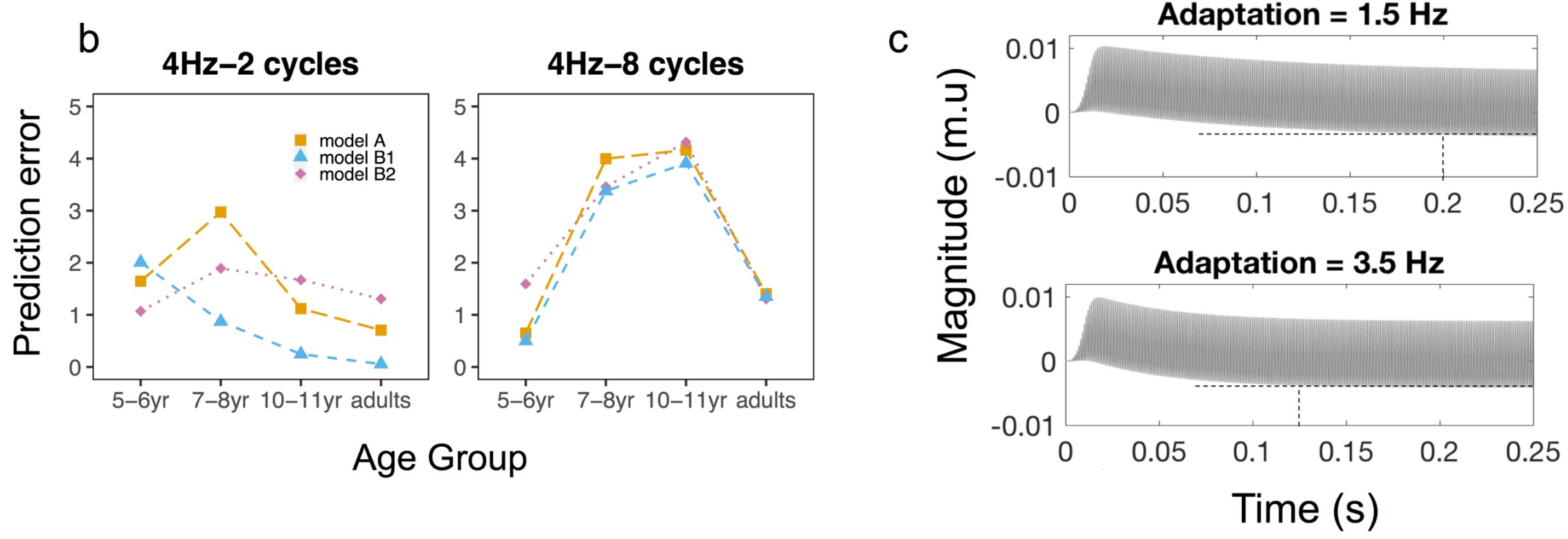
1 Figure 5. a. AMDTs (in $\mathrm{dB}$ ) at $4 \mathrm{~Hz}$ as a function of number of $\mathrm{AM}$ cycles for each age group. Each panel shows real data (green 2 circles) and simulated data (Model A: orange squares; Model B1: blue triangles; Model B2: pink diamonds). Simulations assess 3 changes in internal noise only (Model A), and changes in internal noise and adaptation [Model B1 with stronger adaptation (cutoff 4 frequency of highpass filter $=3.5 \mathrm{~Hz}$ ) vs Model B2 with lower adaptation (cutoff frequency of highpass filter=1.5 Hz)]. b. Model's 5 goodness-of-fit, estimated by the fitting error (absolute difference between observed and simulated data) of each model with 2 cycles 6 (left panel) and 8 cycles (right panel) as a function of age group. c. Output of the adaptation stage of the model, for a cutoff frequency 7 of the highpass filter set at $1.5 \mathrm{~Hz}$ (top panel) and $3.5 \mathrm{~Hz}$ (lower panel): Note that the transient response is stronger (faster decay) for 8 a $3.5-\mathrm{Hz}$ cutoff frequency. 


\subsubsection{Effects of simulating imperfect retention of temporal-envelope information}

As in Wallaert et al. (2017), we simulated poorer processing efficiency for adult listeners by including an additive time-varying memory noise at the output of modulation filters. The standard deviation of this memory noise increased backward in time according to an exponential function using a 1.2-s half life. Memory noise aimed to simulate imperfect retention of temporal-envelope information in each observation interval. This is especially important for long stimuli, and thus, for the $4 \mathrm{~Hz}-8$ cycles condition where the stimulus duration is equal to $2 \mathrm{~s}$. Figure 6.a shows the effect of introducing this exponential memory noise on the predicted AMDTs for the $4-\mathrm{Hz}$ condition using two different SD values for the memory noise (Model C1: 3e-4 m.u., and Model C2: $5 \mathrm{e}-4$ m.u.) but the same half life. It is also important to keep in mind that Models $\mathrm{C} 1$ and $\mathrm{C} 2$ also use the levels of additive internal noise of Model A.

Increasing the level of memory noise does not improve the model's goodnessof-fit for the group of 5-6 years and has little effect for the adults as observed in Figure 6.b. However, manipulating this parameter in the model influences the goodness-offit for the two other age groups and especially with 8 AM cycles. The effects of the memory noise on the internal representation of temporal envelopes are illustrated in Figure 7 for a $4-\mathrm{Hz}$ AM stimulus. Overall, the introduction of memory noise improves the model's goodness-of-fit of AMDTs for the $4-\mathrm{Hz}$ condition between 7 and 11 years. As expected, this improvement is more visible for the longest stimuli, i.e., 8 cycles. This suggests that the fidelity of information retention and the size of the short-term memory buffer influence the model's behavior for the two older child groups. The higher the variance of memory noise, the worse the fidelity of the former memory trace of the temporal envelope. The level of memory noise has little effect on the fit of the model to the results from the youngest children, because they have greater internal noise. Using the higher level of memory noise improves the fit of the model for the older children because the level of the internal noise is lower in these groups. For adults, Model A without additional memory noise provides the best fit of the data. Therefore, memory noise for AM processing seems to decrease over age, and importantly, needs to be appreciated in relation with a decrease in internal noise during childhood. 
a

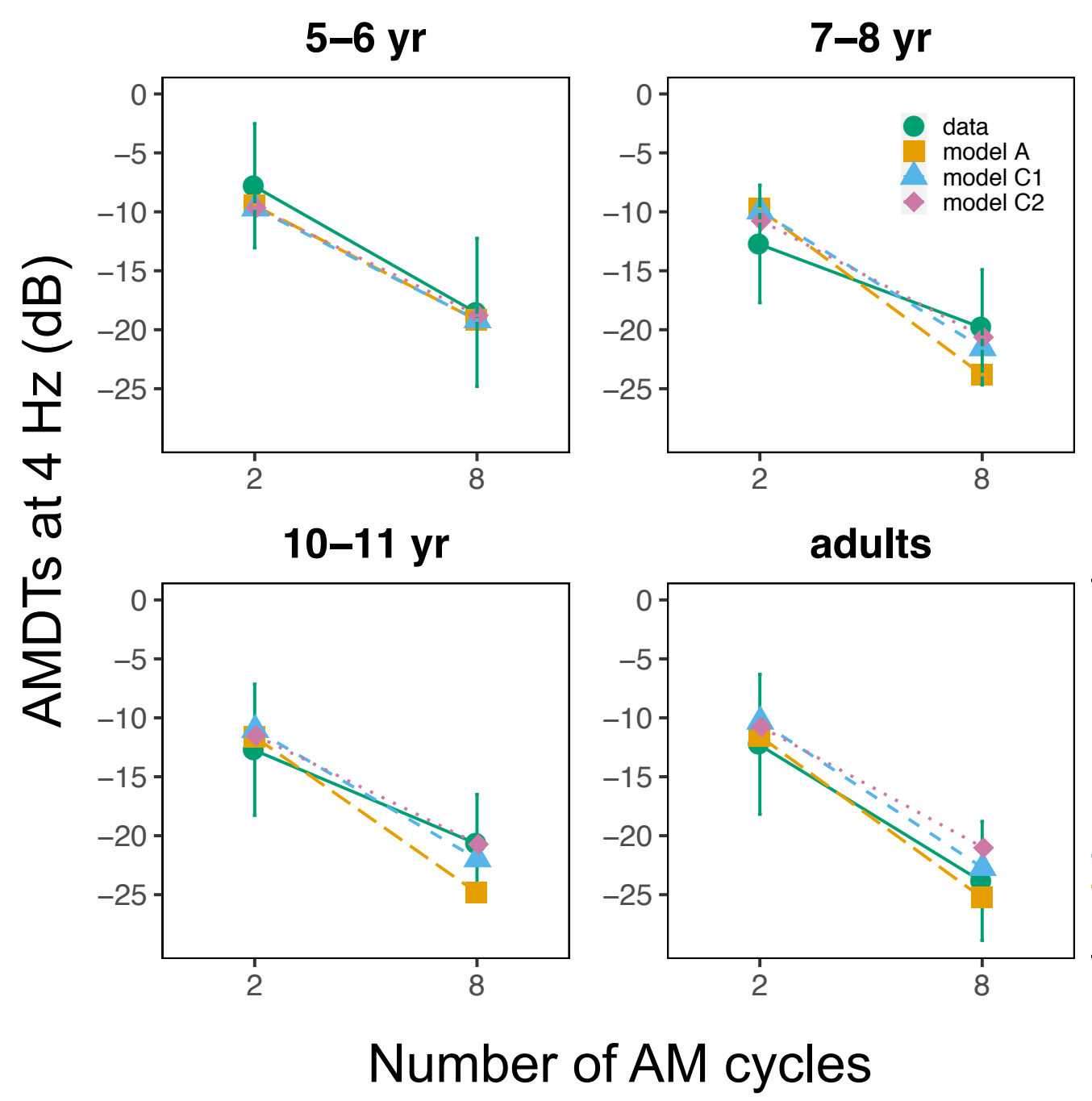

b

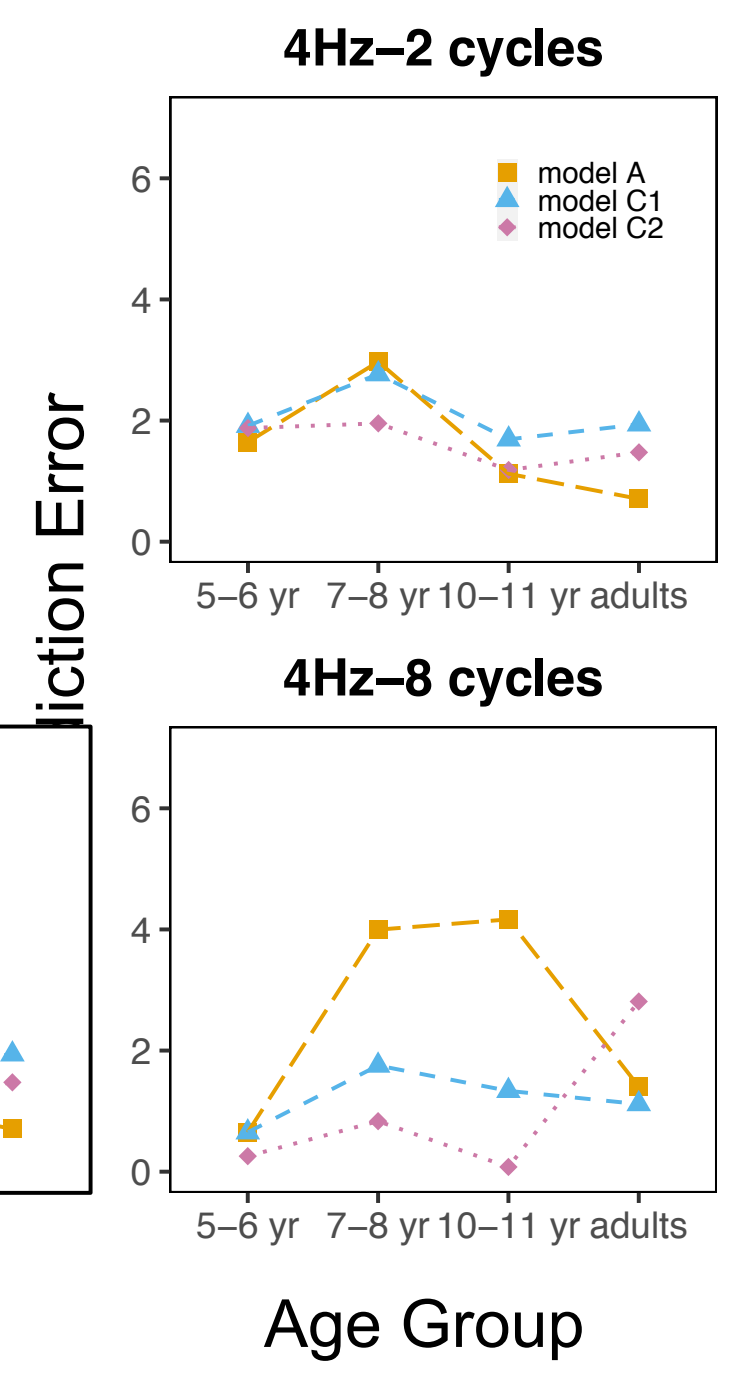


Figure 6. a. AMDTs (in $\mathrm{dB}$ ) at $4 \mathrm{~Hz}$ for each age group as a function of the number of $\mathrm{AM}$ cycles (2 vs 8 cycles) for the real 2 data (solid lines) and the simulated data according to changes in internal noise only (Model A, orange squares and long dashed 3 lines), changes in internal noise and memory noise (Model C1 with low memory noise, blue triangles and dashed lines vs Model C2 4 with higher memory noise, pink diamonds with dotted lines). b. Model's fitting errors (absolute difference between real and simulated 5 data) of each model with 2 cycles (left panel) and 8 cycles (right panel) as a function of age group. 

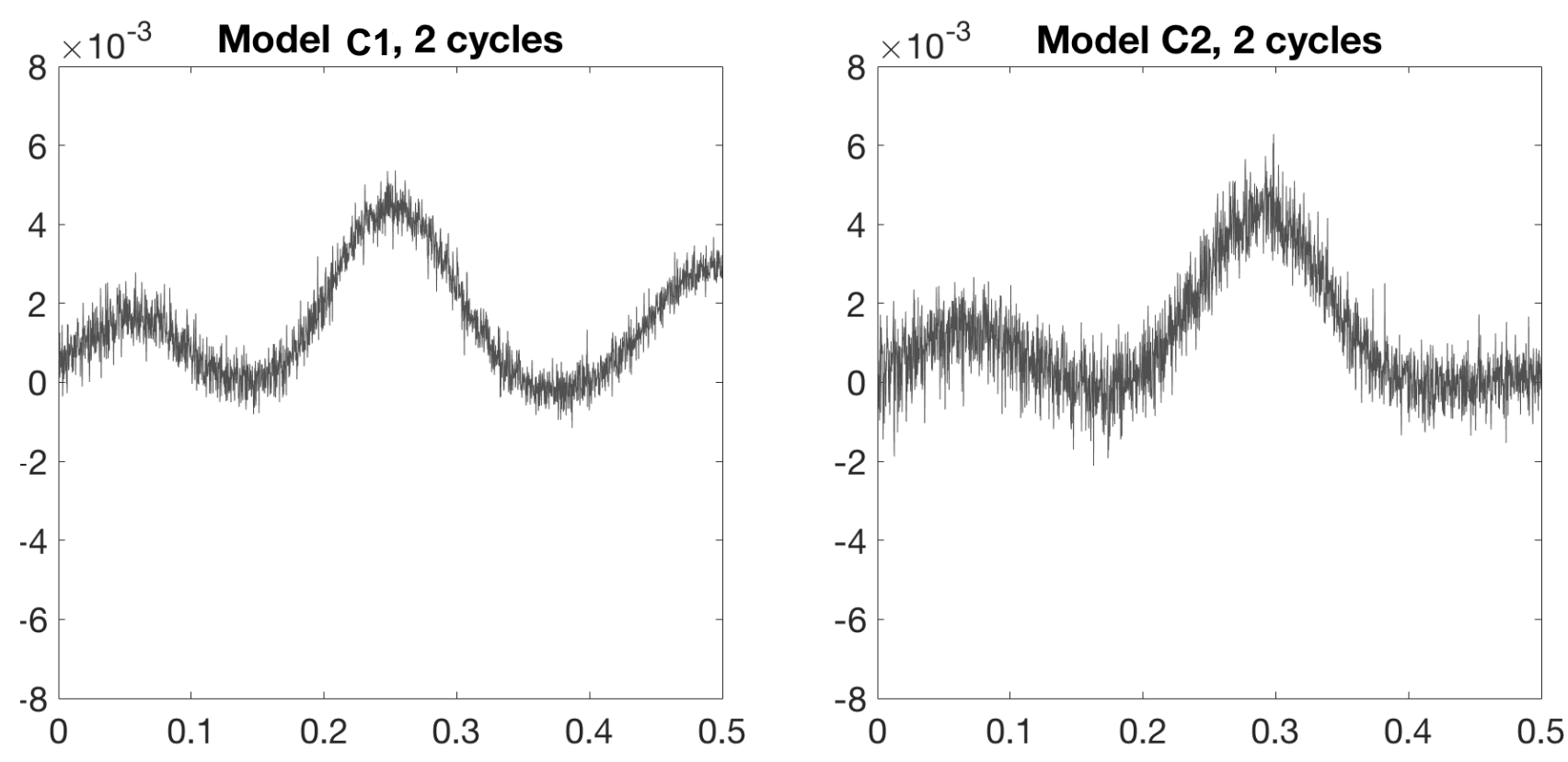

Figure 7. Internal representation of the AM stimulus computed by the model fol two levels of memory noise (low: Mode C1, and high: Model C2) in the conditions $4 \mathrm{~Hz}-2$ cycles and $4 \mathrm{~Hz}-\varepsilon$ cycles.
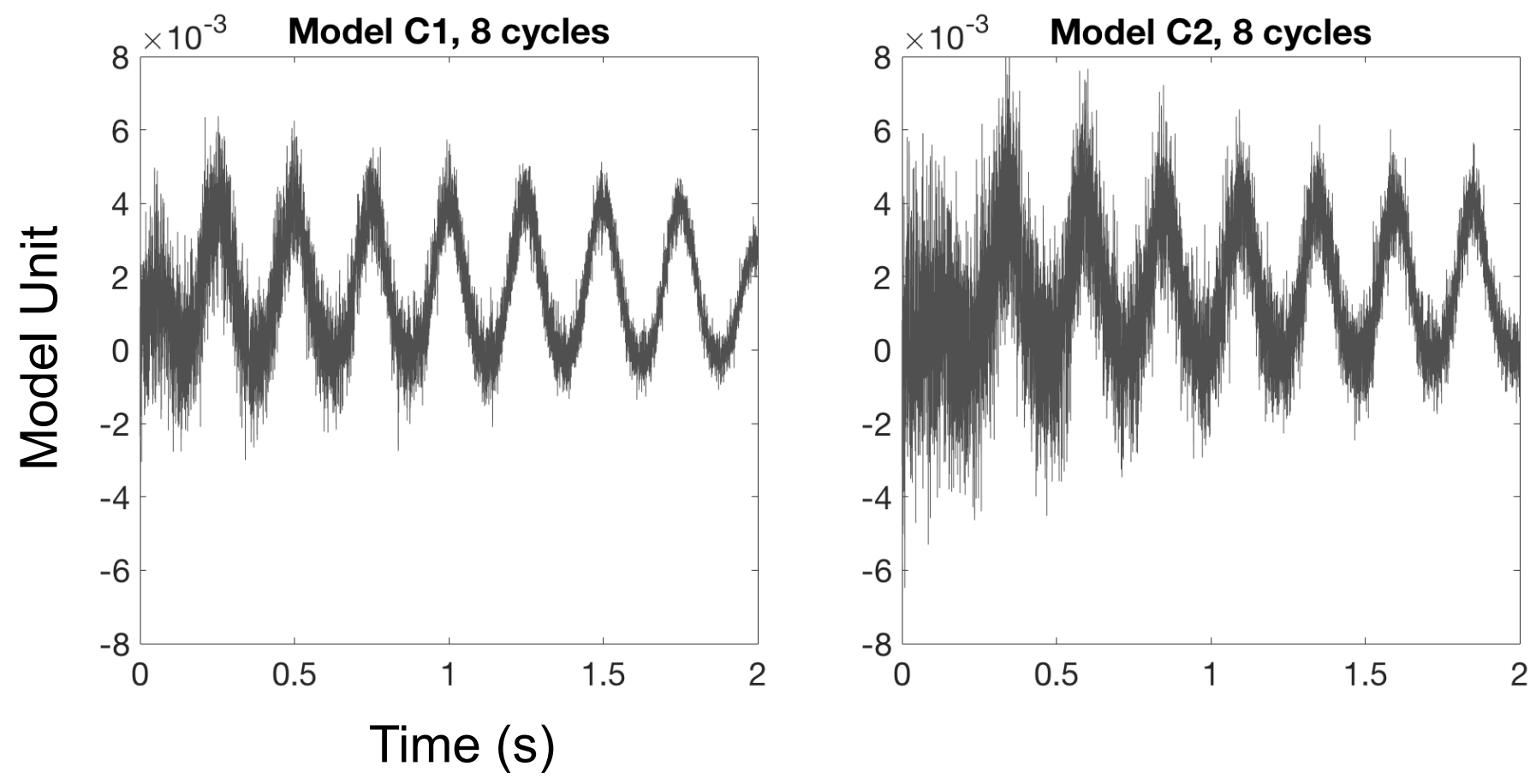


\section{DISCUSSION}

The present study reveals that children spanning the age of 5 to 11 years demonstrate temporal integration for $\mathrm{AM}$ detection. All benefit from an increased number of $A M$ cycles when detecting slow and fast $A M$, and thus demonstrate a capacity to accumulate sensory evidence as to the presence of an AM signal. Surprisingly, greater temporal integration is observed for the youngest children, 5-6 years, compared to the older children, 7-8 years and 10-11 years. This effect results from higher (worse) detection thresholds with 2 AM cycles at 5-6 years but similar thresholds with 8 AM cycles compared to the older children. Overall those results suggest that sensory-processing mechanisms related to temporal integration of AM information are mature by 5 years, but that higher stages of AM processing are still developing.

Importantly, no effect of age between 5 and 11 years is observed with a higher number of AM cycles. The 8-cycle condition represents the only condition where younger children show AM detection thresholds similar to those of older children (see Cabrera et al., 2019). This result suggests that the poorer thresholds usually observed in younger children for AM detection tasks are not due to an overall poorer ability in performing such a psychophysical task. The fact that this effect is observed at two AM rates also suggests that children's responses are independent of the absolute duration of the sounds. If their poorer thresholds were related only to a lack of attention, we should have observed poor thresholds in the shortest-duration condition only, that is, at $32 \mathrm{~Hz}$ and with $2 \mathrm{AM}$ cycles (duration $\sim 62.5 \mathrm{~ms}$ ), and not at $4 \mathrm{~Hz}$ and with 2 cycles (duration $\sim 250 \mathrm{~ms}$ ). The higher (worse) thresholds observed for young children may then be related to their difficulty in processing $\mathrm{AM}$ cues when the modulated target sound shows a small number of AM cycles (as suggested by Peter et al., 2014). This specific result, unrelated to sound duration, may relate to inefficiencies in processing the temporal-envelope information when few cycles are available.

To explore further the stages of AM processing that may develop over age, we used a computational model based on the modulation-filter bank concept simulating peripheral and central constraints in AM processing. The results of the modelling study reveal that, contrary to a previous study on AM masking (Cabrera et al., 2019), the observed data are not well simulated by a simple decrease in the level of internal noise 
1 over age: A progressive decrease in the level of internal noise during childhood 2 predicts better temporal integration with increasing age while the real data show better temporal integration in the youngest group (5-6 years). Additional simulations indicate that changes in short-term adaptation cannot account for the change in temporal integration over age; changing the weight of transients does not influence the model's predictions for 5-6-years or adults, nor does it improve the model's predictions for the other two child groups. Changing the characteristics of adaptation provides a slightly better fit for the 2-cycles condition at 7-8 years but this improvement is not sufficiently large. Thus, children do not seem to weight differently transient responses over age.

Finally, the only aspect of AM processing changing over age that yields better predictions for the 7-8 and 10-11 year groups is imperfect retention of temporalenvelope information, as proposed by Wallaert et al. (2017). In the present model, this constraint is simulated by degrading the temporal-envelopes of incoming signals at the output of modulation filters with an additive, exponential memory noise. Temporalenvelope cues are more masked by memory noise near the onset of the stimulus. Increasing the SD of the memory noise lengthens the initial portion of the stimulus that is most impacted by the memory noise, reducing by the same amount the length of the final portion that makes an effective contribution to decision making. The imperfect retention of temporal-envelope information in each observation interval necessarily has a greater detrimental effect on the detection of the longest stimuli, e.g., at 8 AM cycles. The fact that increasing memory noise only improves model predictions for the 7-8 years and 10-11 years suggests that: 1 ) The level of the additive internal noise at 5-6 years is high enough to dominate any effect of memory noise; 2 ) the level of the memory noise decreases over age, as adults showed significantly better thresholds than the child groups in the 8-cycle condition; 3) AM processing is still not fully mature in late childhood, but this may relate to inefficiency at higher levels of processing, i.e., echoic memory.

Echoic memory, or 'auditory sensory memory', is the ability to retain a sound stimulus right after its occurrence, allowing further processing. This type of memory is described as a pre-attentive phase of $100-300 \mathrm{~ms}$ where auditory information is temporarily stored for further manipulation (Cowan, 1984; Massaro, 1972). So far, 
1 developmental studies have shown that this cognitive function improves throughout 2 childhood and reaches its peak in early adulthood. Evoked-potential investigations have tested the short-term retention and processing of tones, in children vs adults, through oddball paradigms assessing the Mismatch Negativity (MMN) to deviant tones differing in frequency or duration. These studies have globally revealed reduced amplitude and higher latency of the MMN up to 10 years of age, which is thought to reflect the shorter duration and faster decay of the sensory memory trace (see BarthaDoering et al., 2015 for a review). Keller and Cowan (1994) reported comparable results from a 2I-2AFC tone-comparison task, and concluded that the persistence of echoic memory for tones is shorter in children of 6-to-7-year-olds than adults. The clear-cut differences in echoic memory performance between children and adults may explain why the model predictions for AM detection are improved by simulating imperfect retention of temporal-envelope information in childhood.

The dynamics behind the protracted improvement of echoic memory are not yet clear. It has been proposed that a general increase in global processing speed might mediate this process (Ferguson and Bowey, 2005). Neural maturation of specific brain areas may also play a role in improvement of echoic memory. Recent neurophysiological studies in animals suggest that parietal cortex activity relates to the integration of $A M$ information (Yao et al., 2020). When excitatory auditory cortex inputs to parietal cortex are inhibited, this results in reduced temporal integration in gerbils for AM-target stimulations of 100,300 and $600 \mathrm{~ms}$. When parietal cortex is directly deactivated, increased errors at long stimulus durations, of 1 or $2 \mathrm{~s}$, are observed, suggesting that the parietal cortex integrates sensory input from the primary auditory cortex and thus, plays an essential role in an auditory decision task.

Whether parietal cortex is more specifically involved in information retention is still under investigation. Lesion studies suggest that parietal areas are involved in memory judgments through decision making. For instance, patients with parietal lesions show more difficulties in integrating new external cues with internal memory evidence (Dobbins et al., 2012). Finally, the role of prefrontal areas in decision making (Hanks et al., 2015), as well as of auditory areas in auditory short term memory (Scott et al., 2014) need to be specified in such temporal integration tasks. Better knowledge 
1 of the specific development of those cortical regions and their implications for AM processing would help to better understand what stages of processing are still developing through childhood. Computational modelling may also help in future studies to simulate the development of memory processing and of decision making in AM detection.

It is worth noting that our current model did not take into account the potential detrimental effects of memory capacities on the whole sequence of stimuli composing a given trial in the 3AFC procedure. Memory limitations may have not only affected the internal representation (and especially the onset) of each stimulus, but also the whole sequence of stimuli composing a given trial. This may especially be the case when using 8 cycles at $4 \mathrm{~Hz}$, where the whole trial lasts for $7 \mathrm{~s}$. In other words, the internal representation of the first stimulus of a given trial might have been more affected by memory limitations than the internal representation of the second or last stimulus. The current modelling architecture aimed to reproduce aspects of echoic memory in the AM domain, not such effects over the whole trial. For 7-s long sequences of sounds, other aspects of memory may come into play such as working memory. Further work is warranted to investigate such constraints in temporal envelope retention. Still, using a 3AFC procedure, where the position of the target trial is randomized on each trial, this effect should be averaged out in the results. It is also noteworthy that the current model succeeded in reproducing the $4-\mathrm{Hz}$ data without taking account of the temporal structure of the whole trial.

Finally, our model did not take attentional effects into consideration. Wright and Dai (1998) showed that expectations influence AM detection performance (see also (Conroy and Kidd, 2021). To the best of our knowledge, there is no model of auditory modulation processing able to reproduce such uncertainty effects.

\section{v. CONCLUSIONS}

The present study indicated that children from 5 to 11 years, as well as adults, showed an improvement in AM detection thresholds when presented with more AM cycles in the modulated target. Nevertheless, this effect is influenced by age, as the youngest children of 5-6 years displayed the worst thresholds when only 2 cycles were available, irrespective of the AM rate to detect. Furthermore, adults showed the best 
1 thresholds with 8 cycles compared to all age groups. These differences resulted in 2 better temporal integration for AM detection in these two groups compared to the 7-8 3 and $10-11$ years.

The fact that the youngest children were not affected by the absolute duration 5 of the sounds suggested that their poorer thresholds did not relate only to a lack of 6 sustained attention but may relate to inefficiencies, e.g., higher internal noise, in 7 processing the temporal-envelope information when few cycles were available. 8 Moreover, the reduced temporal integration observed between 7 and 11 years pointed 9 towards additional constraints imposed by the limited memory buffer involved in AM 10 processing with longer stimulus durations. Computational modelling confirmed that 11 changes in late processing stages over age (e.g., a reduction of internal noise coupled 12 with a reduction in memory noise) better explained changes in temporal integration 13 between 5 and 11 years than changes in the early stages of AM processing. The 14 model, however, did not account correctly for the data at $32 \mathrm{~Hz}$, probably because of 15 the short duration of the sounds and the dominance of the transient response of the 16 simulated AM filters in this condition. The present study overall suggested that 17 processing efficiency for AM continues to develop late into childhood. 


\section{APPENDIX}

The model structure is similar to that used by Wallaert et al. (2017). The model had the following stages in sequential order:

1. a bank of five gamma-tone filters, one centered at the carrier frequency of the stimulus, and the remaining four centered at 1 and $2 \mathrm{ERB}_{\mathrm{N}}$ above and below the carrier frequency of the stimulus;

2. a 'broken-stick' input-output function for the output of the gamma-tone filter tuned to the carrier frequency of the stimulus; the function is linear up to a knee-point of $30 \mathrm{~dB}$ SPL and compressive (using a power law with an exponent of 0.3) above;

3. half-wave rectification of all five frequency channels;

4. high-pass filtering ( $1^{\text {st }}$ order $3 \mathrm{~dB} /$ oct roll-off, default cut-off value: $2.5 \mathrm{~Hz}$ ) of all frequency channels to simulate the effects of short-term adaptation in the modulation domain (Tchorz and Kollmeier, 1999);

5. the signal of each frequency channel was passed to a filter-bank ( $1^{\text {st }}$ order Butterworth filters) with 10 logarithmically-spaced channels tuned between 2 and $120 \mathrm{~Hz}$ (Moore et al., 2009), each with a $Q$ factor of 1 (Ewert and Dau, 2000) to decompose the modulations of the processed signals, producing 50 channels;

6. the model preserved temporal-envelope phase at the output of modulation filters tuned below $6 \mathrm{~Hz}$. The model discarded the envelope phase for channels above 6 $\mathrm{Hz}$ by passing only the absolute magnitude of the Hilbert transform of the outputs to the following stage;

7. two independent Gaussian noises (an 'additive' and a 'memory' noise) were added consecutively to the output of all 50 channels; the first type of noise ("additive noise") had a constant standard deviation (SD) (Dau et al., 1997a); the second type of noise ("memory noise") was additive like the first one, but had an SD which was multiplied by an exponential decay function to model echoic-memory limitation; the addition of this "memory noise" resulted in a weaker representation of the earlier part of the signal than the later and reduced temporal integration of envelope cues (Ardoint et al., 2008; Wallaert et al., 2017), disrupting the representation of longer duration stimuli more than shorter duration stimuli. The decay time constant was fixed at $1.4 \mathrm{~s}$; 
1 8. the final decision stage was based on a template matching process (Dau et al., 1997a). The model generated an "internal template" at the start of each staircase with the modulation depth set at the starting value and without any internal noise. The internal template was calculated as the difference between the internal representations of the target and reference stimuli, channel by channel. On each trial, the target and reference stimulus intervals were cross-correlated (channel by channel) with the template. The lags used in the cross-correlation were restricted to \pm 1 target modulation cycle. The interval with the largest cross-correlation coefficient (summed across channels) was selected by the model.

\section{ACKNOWLEDGMENTS}

This work was supported by the European Union's Horizon 2020 research and innovation programme under the Marie Sklodowska-Curie Grant Agreement No. 659204 held at the Department of Speech, Hearing and Phonetic Sciences, UCL and by Grant No. ANR-17-CE28-008 awarded to L.C. C.L. was also supported by Grants Nos. ANR-11-0001-02 PSL and ANR-10-LABX-0087. The authors wish to thank Gianna Li and Lucia Sanz for help with testing at schools, as well as Emmanuel Ponsot for insightful discussions on the results. Finally, the authors thank all the participants and schools who participated in the study.

\section{REFERENCES}

Ardoint, M., Lorenzi, C., Pressnitzer, D., Gorea, A., 2008. Investigation of perceptual constancy in the temporal-envelope domain. J. Acoust. Soc. Am. 123, 15911601. https://doi.org/10.1121/1.2836782

Bacon, S.P., Grantham, D.W., 1989. Modulation masking: Effects of modulation frequency, depth, and phase. J. Acoust. Soc. Am. 85, 2575-2580.

Baker, R.J., Rosen, S., 2001. Evaluation of maximum-likelihood threshold estimation with tone-in-noise masking. Br. J. Audiol. 35, 43-52.

Bartha-Doering, L., Deuster, D., Giordano, V., am Zehnhoff-Dinnesen, A., Dobel, C., 2015. A systematic review of the mismatch negativity as an index for auditory sensory memory: From basic research to clinical and developmental perspectives. Psychophysiology 52, 1115-1130. 
1 Biberger, T., Ewert, S.D., 2016. Envelope and intensity based prediction of psychoacoustic masking and speech intelligibility. J. Acoust. Soc. Am. 140, 1023-1038.

Buss, E., Lorenzi, C., Cabrera, L., Leibold, L.J., Grose, J.H., 2019. Amplitude modulation detection and modulation masking in school-age children and adults. J. Acoust. Soc. Am. 145, 2565-2575.

Cabrera, L., Varnet, L., Buss, E., Rosen, S., Lorenzi, C., 2019. Development of temporal auditory processing in childhood: Changes in efficiency rather than temporal-modulation selectivity. J. Acoust. Soc. Am. 146. https://doi.org/https://doi.org/10.1121/1.5128324

Conroy, C., Kidd Jr, G., 2021. Uncertainty about amplitude eliminates negative masking in a pure-tone amplitude discrimination experiment. JASA Express Lett. 1, 14403.

Cowan, N., 1984. On short and long auditory stores. Psychol. Bull. 96, 341.

Dau, T., Kollmeier, B., Kohlrausch, A., 1997a. Modeling auditory processing of amplitude modulation. I. Detection and masking with narrow-band carriers. J. Acoust. Soc. Am. 102, 2892-2905.

Dau, T., Kollmeier, B., Kohlrausch, A., 1997b. Modeling auditory processing of amplitude modulation. II. Spectral and temporal integration. J. Acoust. Soc. Am. 102, 2906-2919.

Dobbins, I.G., Jaeger, A., Studer, B., Simons, J.S., 2012. Use of explicit memory cues following parietal lobe lesions. Neuropsychologia 50, 2992-3003.

Drullman, R., Festen, J.M., Plomp, R., 1994a. Effect of temporal envelope smearing on speech reception. J. Acoust. Soc. Am. 95, 1053.

Drullman, R., Festen, J.M., Plomp, R., 1994b. Effect of reducing slow temporal modulations on speech reception. J. Acoust. Soc. Am. 95, 2670.

Edwards, D.R., Lee, J., Andrews, J., Wong, A., 2008. Contribution of onset/offset information of modulation to amplitude modulation depth discrimination. J. Acoust. Soc. Am. 123, EL111-EL115.

Ewert, S.D., Dau, T., 2000. Characterizing frequency selectivity for envelope fluctuations. J. Acoust. Soc. Am. 108, 1181-1196. 
1 Ferguson, A.N., Bowey, J.A., 2005. Global processing speed as a mediator of developmental changes in children's auditory memory span. J. Exp. Child Psychol. 91, 89-112.

Goswami, U., Thomson, J., Richardson, U., Stainthorp, R., Hughes, D., Rosen, S., Scott, S.K., 2002. Amplitude envelope onsets and developmental dyslexia: A new hypothesis. Proc. Natl. Acad. Sci. 99, 10911-10916.

Hall, J.W., 3rd, Grose, J.H., 1994. Development of temporal resolution in children as measured by the temporal modulation transfer function. J. Acoust. Soc. Am. 96, 150-154.

Hanks, T.D., Kopec, C.D., Brunton, B.W., Duan, C.A., Erlich, J.C., Brody, C.D., 2015. Distinct relationships of parietal and prefrontal cortices to evidence accumulation. Nature 520, 220-223.

Hoaglin, D.C., Iglewicz, B., 1987. Fine-tuning some resistant rules for outlier labeling. J. Am. Stat. Assoc. 82, 1147-1149.

Houtgast, T., 1989. Frequency selectivity in amplitude-modulation detection. J. Acoust. Soc. Am. 85, 1676-1680.

Keller, T.A., Cowan, N., 1994. Developmental increase in the duration of memory for tone pitch. Dev. Psychol. 30, 855.

King, A., Varnet, L., Lorenzi, C., 2019. Accounting for masking of frequency modulation by amplitude modulation with the modulation filter-bank concept. J. Acoust. Soc. Am. 145, 2277-2293.

Lee, J., Bacon, S.P., 1997. Amplitude modulation depth discrimination of a sinusoidal carrier: Effect of stimulus duration. J. Acoust. Soc. Am. 101, 36883693.

Levitt, H., 1971. Transformed up-down methods in psychoacoustics. J. Acoust. Soc. Am. 49, 467-477.

Liégeois-Chauvel, C., Lorenzi, C., Trébuchon, A., Régis, J., Chauvel, P., 2004. Temporal envelope processing in the human left and right auditory cortices. Cereb. Cortex 14, 731-740.

Lorenzi, C., Dumont, A., Fullgrabe, C., 2000. Use of temporal envelope cues by children with developmental dyslexia. J. Speech Lang. Hear. Res. 43, 1367. 
1 Massaro, D.W., 1972. Preperceptual images, processing time, and perceptual units in auditory perception. Psychol. Rev. 79, 124.

Moore, B.C., Füllgrabe, C., Sek, A., 2009. Estimation of the center frequency of the highest modulation filter. J. Acoust. Soc. Am. 125, 1075-1081.

Peter, V., Wong, K., Narne, V.K., Sharma, M., Purdy, S.C., McMahon, C., 2014. Assessing spectral and temporal processing in children and adults using temporal modulation transfer function (TMTF), iterated ripple noise (IRN) perception, and spectral ripple discrimination (SRD). J. Am. Acad. Audiol. 25, 210-218.

Rosen, S., 1992. Temporal information in speech: acoustic, auditory and linguistic aspects. Philos. Trans. R. Soc. Lond. B. Biol. Sci. 336, 367-373. https://doi.org/10.1098/rstb.1992.0070

Scott, B.H., Mishkin, M., Yin, P., 2014. Neural correlates of auditory short-term memory in rostral superior temporal cortex. Curr. Biol. 24, 2767-2775.

Sheft, S., Yost, W.A., 1990. Temporal integration in amplitude modulation detection. J. Acoust. Soc. Am. 88, 796-805.

Tchorz, J., Kollmeier, B., 1999. A model of auditory perception as front end for automatic speech recognition. J. Acoust. Soc. Am. 106, 2040-2050.

Varnet, L., Ortiz-Barajas, M.C., Erra, R.G., Gervain, J., Lorenzi, C., 2017. A crosslinguistic study of speech modulation spectra. J. Acoust. Soc. Am. 142, 19761989.

Viemeister, N.F., 1979. Temporal modulation transfer functions based upon modulation thresholds. J. Acoust. Soc. Am. 66, 1364.

Viemeister, N.F., Wakefield, G.H., 1991. Temporal integration and multiple looks. J. Acoust. Soc. Am. 90, 858-865.

Walker, B.A., Gerhards, C.M., Werner, L.A., Horn, D.L., 2019. Amplitude modulation detection and temporal modulation cutoff frequency in normal hearing infants. J. Acoust. Soc. Am. 145, 3667-3674.

Wallaert, N., Moore, B.C., Ewert, S.D., Lorenzi, C., 2017. Sensorineural hearing loss enhances auditory sensitivity and temporal integration for amplitude modulation. J. Acoust. Soc. Am. 141, 971-980. 
1 Wallaert, N., Moore, B.C., Lorenzi, C., 2016. Comparing the effects of age on

2

3

4

5

6

7

8

9 amplitude modulation and frequency modulation detection. J. Acoust. Soc. Am. 139, 3088-3096.

Wallaert, N., Varnet, L., Moore, B.C., Lorenzi, C., 2018. Sensorineural hearing loss impairs sensitivity but spares temporal integration for detection of frequency modulation. J. Acoust. Soc. Am. 144, 720-733.

Witton, C., Stein, J.F., Stoodley, C.J., Rosner, B.S., Talcott, J.B., 2002. Separate influences of acoustic AM and FM sensitivity on the phonological decoding skills of impaired and normal readers. J. Cogn. Neurosci. 14, 866-874.

Wright, B.A., Dai, H., 1998. Detection of sinusoidal amplitude modulation at unexpected rates. J. Acoust. Soc. Am. 104, 2991-2996.

Yao, J.D., Gimoto, J., Constantinople, C.M., Sanes, D.H., 2020. Parietal cortex is required for the integration of acoustic evidence. Curr. Biol. 30, 3293-3303. 